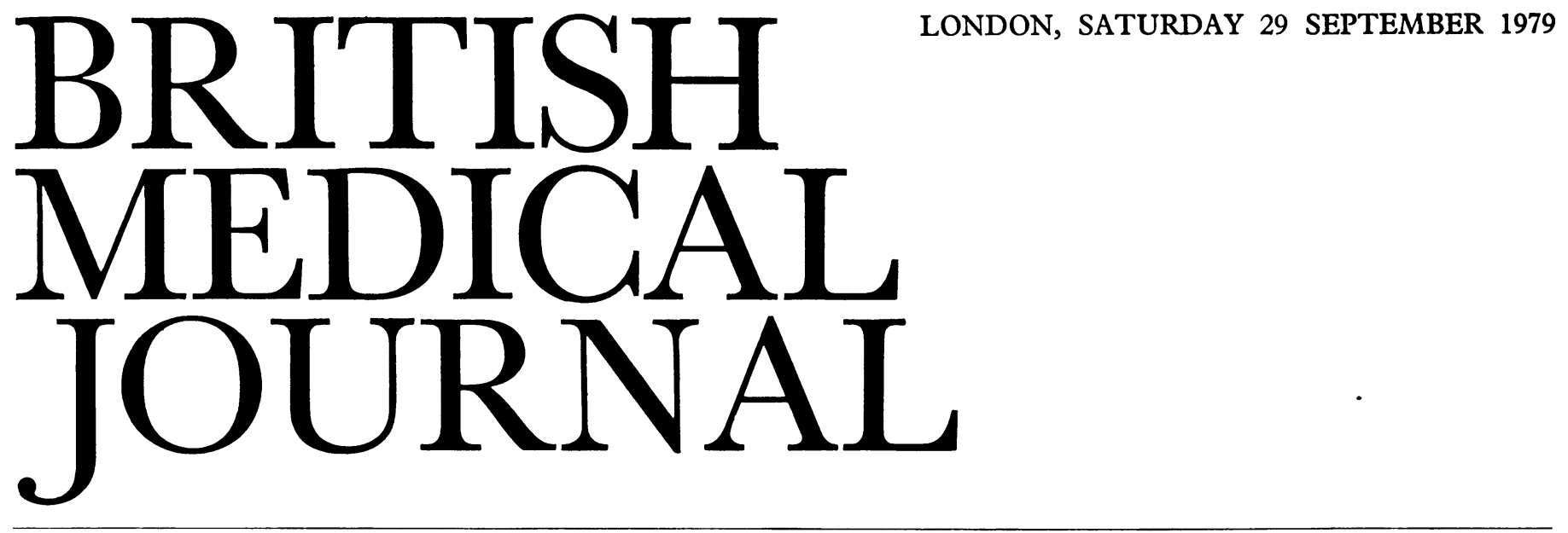

\title{
The concept of disease
}

There is no concept in medicine more fundamental than that of disease, yet few doctors ever give a moment's thought to its precise definition. Most of the time they do not need to, but there are some circumstances in which this curious neglect causes insoluble problems. As Scadding ${ }^{1}$ has pointed out, several of the issues chest physicians argue about inconclusively are really unrecognised disputes about the definining characteristics of diseases like chronic bronchitis, emphysema, and asthma; the same is true of arguments about the relation between rheumatoid arthritis and the collagen diseases. Mental illness provides a particularly fertile source of controversy; the inability of either psychiatrists or laymen to agree whether homosexuality, alcoholism, and psychopathy are correctly regarded as diseases, or indeed whether there is any such thing as mental illness, is basically due to a failure to agree, or even to consider, what disease itself is. ${ }^{2}$

The concept of disease probably arose historically as an explanation for suffering or incapacity developing in the absence of obvious injury. Diseases were entities with a shadowy metaphysical existence of their own that were responsible for the symptoms of the people they afflicted. In contrast to this traditional essentialist or realist outlook is the nominalist view of the contemporary medical scientist, who regards diseases as arbitrary concepts, a convenient name given to a specified group of phenomena and liable at any time to be adjusted or discarded. A realist assumes that Gull discovered myxoedema. A nominalist insists that he merely introduced the term myxoedema, that for 80 years it was widely used because it helped make sense of clinical observations, and that it was eventually discarded when the more useful concept of hypothyroidism was introduced.

In an attempt to tackle what they rightly regard as an important and neglected issue without getting bogged down in sterile semantic disputes, Professors Moran Campbell and $\mathrm{J}$ G Scadding have investigated how the term disease is used in practice. They read a list of 38 terms such as tuberculosis, schizophrenia, drowning, and starvation to groups of hospital physicians, general practitioners, secondary school children, and non-medical university teachers and persuaded these audiences to commit themselves to deciding quickly whether or not each of the 38 phenomena was a disease. The results of this simple but unusual exercise are reported at $\mathrm{p} 757$. Everyone regarded infections like malaria and syphilis as diseases, but the doctors had a consistent tendency to regard a wider range of phenomena as disease than either of the lay groups. General practitioners also had a broader usage than hospital physicians. The differences between doctors and laymen were greatest for conditions with an obvious physical cause, like fractured skull, tennis elbow, and lead poisoning, confirming that for most people disease is still basically an explanatory concept invoked only when there is no obvious cause for the disability. Another influential factor was the need for medical intervention, for ratings of the doctor's role in diagnosis and treatment correlated highly in both medical and lay audiences with the main "disease connotation" scores. The simplest explanation for the greater willingness of general practitioners than of hospital physicians to regard such conditions as alcoholism, hypertension, and senility as diseases is their customary acceptance of a wider therapeutic role. Social scientists have maintained for some time that disease is a sociopolitical concept masquerading as a biomedical one, and that when doctors or other people label something as a disease all they are really saying is that they regard medical intervention as appropriate. ${ }^{3-5}$ Probably few doctors would agree, but these results can be construed as giving at least partial support to this view.

Scadding and Moran Campbell draw an analogy between the formal scientific and the everyday uses of the term disease and those of terms like force and power in the physical sciences. In both cases the vagueness of colloquial usage is unimportant provided that the scientific usage is consistent and unambiguous. They argue that for scientific purposes a nominalist concept of disease should be obligatory, and to emphasise this suggest that Scadding's 1967 definition $^{1}$ should be reworded slightly as follows: "In medical discourse, the name of a disease refers to the sum of the abnormal phenomena displayed by a group of living organisms in association with a specified common characteristic or set of characteristics by which they differ from the norm for their species in such a way as to place them at a biological disadvantage."

For some diseases (migraine, schizophrenia) the defining characteristic is their clinical syndrome, for others it is a structural abnormality (mitral stenosis, sarcoidosis), or a functional abnormality (hypothyroidism, myasthenia gravis) or aetiological agent (typhoid, syphilis). For some the defining characteristic takes in more than one of these categories: tabes dorsalis, for example, implies both particular anatomical changes and a syphilitic aetiology. Initially, the defining characteristic of most diseases is their clinical syndrome, but as knowledge of their causation accumulates there is usually a 
shift to one of the other modes of definition, as in the transition from myxoedema to hypothyroidism, or from Down's syndrome to trisomy 21 .

Unfortunately, many doctors remain blissfully unaware of this logical structure and continue to be influenced by outmoded realist assumptions. One still hears eminent physicians say, " $X$ isn't a disease, it's only a syndrome"; psychiatrists still say, "I'm sure he's a schizophrenic even though he doesn't yet show any of the typical symptoms"; and chest physicians still argue whether a patient has chronic bronchitis (a clinical syndrome) or emphysema (a structural abnormality). As an empirical discipline medicine has always been suspicious of anything that smacks of philosophy, but this attitude may be a luxury we cannot afford where our own most fundamental concept is concerned. If for no other reason, Moran Campbell and Scadding's study is welcome as evidence that sociologists are no longer the only people with an interest in the meaning of the term disease.

\footnotetext{
1 Scadding, J G, Lancet, 1967, 2, 877.

2 Kendell, R E, British fournal of Psychiatry, 1975, 127, 305.

${ }^{3}$ Linder, R, Perceptual and Motor Skills, 1965, 20, suppl p 1081.

4 Sedgwick, P, Hastings Center Studies, 1973, 3, 19.

5 Engelhardt, H T, in Evaluation and Explanation in the Biomedical Sciences, ed H T Engelhardt and S F Spicker, p 137. Dordrecht, Reidel, 1975.
}

\section{Bed bugs, insects, and hepatitis $B$}

Hepatitis B virus may be transmitted in two main ways. The first is by blood and some plasma derivatives, and by any procedure in which the skin or mucosa is penetrated by inadequately sterilised contaminated needles and instruments. Known modes of transmission of hepatitis B include tattooing, acupuncture, piercing of the ear and nose, scarification, ritual operations, and blood letting. The second main method of spread occurs non-parenterally, by intimate contact and by the sexual route. Both of these latter possibilities have been recognised more recently, but we now know that this list does not exhaust the epidemiological propensities of this infection: is, for instance, hepatitis B spread by mosquitoes and other blood-sucking arthropods, particularly in hot climates? This possibility has been studied for several years, but the results have been conflicting. ${ }^{1}$ Hepatitis B surface antigen, a marker of the virus, ${ }^{2}$ has been detected in several species of mosquitoes trapped in the wild or fed artificially on infected blood. Even so, no convincing evidence has been obtained of either replication of the virus or persistence of the antigen in the insect.

Bed bugs, on the other hand, live more intimately with man than mosquitoes, feed on blood, and could transfer blood and hepatitis B virus from one occupant of a bed to another. Indeed, hepatitis B surface antigen was detected in one of 18 pools of engorged bed bugs (species Cimex hemipterus) collected from brothels in the Ivory Coast. ${ }^{3}$ In a laboratory study two species of bed bugs-the common bed bug, $C$ lectularius, and Rhodinus prolixus from South America-were fed artificially on blood from a patient with acute hepatitis B. ${ }^{4}$ The surface antigen remained detectable in the bugs for over four weeks, and juvenile bugs fed on the antigen when in the fourth or fifth instar stage still retained it after moulting-the time when bugs usually start to search for a host and to refeed. In another study, ${ }^{5}$ bed bugs of the species $C$ hemipterus were collected on several separate occasions from bedding in village huts in Senegal. Hepatitis B surface antigen was detected in engorged and unengorged nymph and adult bed bugs, as well as in bugs kept alive without a blood meal for 30 days. Moreover, e antigen (a marker of infectivity of hepatitis B virus ${ }^{2}$ ) was found in one engorged and one unengorged bed bug.

Hence we might reasonably deduce that bed bugs feeding on the occupants of the same bed could increase the risk of hepatitis B infection. Blood-sucking bed bugs can regurgitate virus, and it might be present in their saliva; while two other modes of transmission of the virus might be by killing the insect during feeding, and by faecal extrusion of the unaltered virus by the bug after a meal of blood. By themselves these observations are insufficient evidence for accepting the bed bug as a vector of hepatitis B virus, but Jupp and McElligott ${ }^{6}$ have now taken the story a step further. A colony of $C$ lectularius was fed on blood containing hepatitis B surface antigen. Again, there was no evidence that the virus replicated in the bugs: the antigen persisted after one moult only (transstadial transmission) and it was not transmitted transovarially. Nevertheless, antigen was transmitted by adult bugs through a membrane into three out of 35 cannisters of antigen-negative blood and, as judged by the acquisition of hepatitis B surface antibody, to a rabbit by adult bugs and to two out of 10 guinea-pigs on which antigen-positive fourth and fifth nymphal instars had fed.

These findings indicate that bed bugs can transmit hepatitis B mechanically to non-permissive hosts, and it is reasonable to assume that transmission rates to susceptible primates might be high. Hence the question of transmission of hepatitis $\mathbf{B}$ by blood-sucking insects merits further investigation, especially since we could at least control this type of spread of this important infection.

1 Viral Hepatitis, World Health Organisation Technical Report Series, No 570. Geneva, World Health Organisation, 1975.

2 Zuckerman, A J, British Medical fournal, 1979, $2,84$.

3 Brotman, B, Prince, A M, and Godfrey, H R, Lancet, 1973, 1, 1305.

4 Newkirk, M M, Downe, A E R, and Simon, J B, Gastroenterology, 1975, 69, 982 .

5 Wills, W, et al, Lancet, 1977, 2, 217.

6 Jupp, P G, and McElligott, S E, South African Medical Fournal, 1979, 2, 54.

\section{Treatment of advanced prostatic carcinoma}

Carcinoma of the prostate commonly spreads to bone and lymphatics. If metastases are sought diligently they will sometimes be found in men whose local disease is apparently at an early stage, and almost always in those in whom it is advanced. ${ }^{2}$ The treatment of the latter is therefore frequently palliative.

In the patient with advanced disease obstruction to the urinary outflow by the primary tumour is easily dealt with by transurethral resection and pain from localised bone metastases is often effectively controlled by radiotherapy. Thereafter we have few guidelines in planning long-term treatment of metastases, partly because of the generally slow progression of untreated disease and partly because probably as many men die with prostatic cancer as from it. Nevertheless, $80-90 \%$ of all patients with prostatic carcinoma will respond, at least temporarily, to treatment aimed at limiting the production of androgens or at stopping 